\title{
Fidélisation, communication et association
}

\section{Céline Nguyen}

\section{OpenEdition}

Journals

Édition électronique

URL : http://journals.openedition.org/communicationorganisation/3249

DOI : 10.4000/communicationorganisation.3249

ISSN : 1775-3546

\section{Éditeur}

Presses universitaires de Bordeaux

\section{Édition imprimée}

Date de publication : 1 juin 2005

ISSN : 1168-5549

\section{Référence électronique}

Céline Nguyen, «Fidélisation, communication et association », Communication et organisation [En ligne], 27 | 2005, mis en ligne le 07 juin 2012, consulté le 02 mai 2019. URL : http:// journals.openedition.org/communicationorganisation/3249; DOI : 10.4000/ communicationorganisation.3249

Ce document a été généré automatiquement le 2 mai 2019.

(c) Presses universitaires de Bordeaux 


\title{
Fidélisation, communication et association
}

\author{
Céline Nguyen
}

1 Les membres des associations d'amis de musées ont pour habitude de se définir comme un public organisé et fidèle au musée. Se démarquant ainsi du public « ordinaire » par son intérêt pour le musée, les associations sont supposées lui venir en aide. Le lexique commun indique qu'un ami est d'ailleurs fidèle par nature, il est celui en qui on a confiance. Dans les faits, les adhérents de ces associations bénéficient en retour de l'entrée gratuite au musée ou des tarifs préférentiels.

2 Mais la manière dont ces associations communiquent indique que la fidélité la plus évidente n'est pas celle qui relie les membres à l'institution musée mais aux activités organisées par l'association : visites, voyages, conférences, ateliers, etc.

3 Cette réflexion ne s'attache pas à décrire les différentes stratégies de fidélisation établies et assumées par les associations d'amis de musées mais à se demander dans quelle mesure l'analyse de la communication institutionnelle reflète, ou non, une intention de fidéliser les membres à ces activités culturelles. Nous définissons la fidélisation comme une stratégie qui vise à développer la "consommation» des clients ou à éviter qu'ils s'en aillent (Bénavent, Meyer-Waarden, 2004). Par analogie, nous nous demanderons si les outils de communication n'ont pas pour fonction (inconsciente ou non) d'influencer les membres à renouveler leur adhésion et surtout à participer aux activités culturelles.

\section{Fidélisation : enjeux et moyens de communication}

4 Avant de répondre à la question de l'existence ou non d'une intention de fidéliser, voyons quels sont les enjeux généraux liés à la fidélisation des adhérents associatifs. 


\section{Les enjeux de la fidélisation à l'association}

5 Les enjeux de la fidélisation en milieu associatif sont de plusieurs ordres. Il s'agit tout d'abord de conserver un nombre suffisant d'adhérents. Dans le cas des amis de musée cela peut avoir son importance puisque la cotisation sert en partie à soutenir le musée dans ses acquisitions. Mais plus largement, le nombre d'adhérents est un bon indicateur de l'activité et de l'attractivité de l'association et entre de ce fait en ligne de compte dans la reconnaissance par les pouvoirs publics.

6 Mais fidéliser les adhérents relève aussi d'une stratégie organisationnelle, puisque chaque association a besoin d'un vivier d'adhérents actifs (Barthélémy, 2000) sans qui l'organisation ne pourrait rester pérenne.

7 Dans le cas des associations d'amis de musées, nous pensons que l'enjeu tel qu'il est médiatisé est plutôt de l'ordre du maintien et de l'accroissement du nombre d'adhérents.

\section{Un engagement dans la communication}

8 Nous avons construit cette réflexion à partir de l'analyse d'un corpus de supports de communication écrite. A leur origine, on retrouve un groupe d'associations basées en Rhône-Alpes ${ }^{1}$. Nous avions remarqué dans une thèse que ces associations possédaient une activité communicationnelle interne et externe plus développée que les autres associations d'amis de musées (Thévenard-Nguyen, 2002)². Nous leur avions reconnu quelques «talents de communication" sans pour autant pouvoir qualifier cette communication institutionnelle de professionnelle. Néanmoins, le recours à une agence de communication, la qualité formelle des documents, la diversité des supports, la régularité de leur parution, etc. témoignent d'efforts remarquables en milieu associatif.

Une cohérence institutionnelle se dégage de ce groupe d'associations : créées à partir des années soixante-dix, elles relèvent généralement de musées des beaux-arts ou d'histoire et se situent dans des villes de préfecture. Elles possèdent un nombre d'adhérents assez élevé ${ }^{3}$ et enfin, adhèrent à la Fédération Française des Sociétés d'Amis de Musées ${ }^{4}$.

10 C'est ce «professionnalisme communicationnel » qui a enclenché cette réflexion sur la fidélisation et par conséquent, le choix et l'étude de ce corpus. Dans quelle mesure les choix de communication sont révélateurs d'une intention de fidéliser les membres de l'association ? L'intensité communicationnelle indique-t-elle une intention de fidéliser?

\section{L'analyse des programmes et bulletins}

11 Le corpus de travail rassemble des outils de communication écrite diffusés par les associations entre les années quatre-vingt-dix et 2000-2001. Il est constitué d'outils représentatifs de la communication associative: périodiques (bulletins, lettres d'information, revues), programmes, communication événementielle (plaquette d'anniversaire) ou plaquette de présentation (document d'appel). Au moment de la collecte, rares étaient ces associations dotées d'un site Internet ${ }^{5}$.

Le programme est mis à disposition des visiteurs du musée ou de l'office de tourisme même s'il est avant tout à destination des membres de l'association. Il en va de même pour la lettre d'information ou le bulletin: bien que disponibles dans les bibliothèques 
spécialisées et/ou locales, ils sont avant tout lus par les membres de l'association puisqu'il y est généralement question d'activités, de projet, de vie associative. Ces documents renforcent le lien entre les membres mais aussi entre les membres et la structure associative.

\section{Communication et fidélisation}

Le rôle de la communication dans une stratégie de fidélisation est primordial.

\section{En entreprise}

Darpy et Volle (2003) indiquent que les facteurs explicatifs de la fidélité à la marque sont les suivants : l'inertie des comportements, la sensibilité à la qualité des marques, la vraie préférence pour un produit, la faible quantité de concurrents, une satisfaction élevée, les liens solides avec le personnel, le coût élevé du changement et le degré de risque perçu. Bénavent et Meyer-Waarden (2004) affirment en écho que la fidélisation est liée à un sentiment de confiance, d'engagement et d'attachement.

Parmi ces facteurs, certains prennent appui sur les outils de communication. D'ailleurs, les spécialistes s'accordent à dire que la communication est l'un des ingrédients majeurs de la recette de la fidélisation (Darpy et Volle, 2003). A un premier niveau, les outils informent les fidèles des modalités de fonctionnement du programme de fidélisation.

En amont, les outils de communication ont pour fonction de donner envie, de convaincre. Selon Noyé, "la communication doit faire une promesse» (2004:36) et affiche la valeur ajoutée à l'origine de la consommation durable du produit. C'est ainsi que la communication "peut renforcer l'importance de certains attributs sur lesquels le produit est particulièrement performant. Ainsi Bouygues Telecom met l'accent sur la qualité du son » (Darpy et Volle, $2003: 176$ ). Nous voyons bien comment la communication peut participer à une stratégie de fidélisation: en suscitant l'engagement, l'attachement et la confiance, en jouant sur la satisfaction, la qualité ou le risque qu'il y aurait à ne plus être client de telle ou telle entreprise ou en suscitant la complicité entre le client et la marque.

Les outils aident aussi à personnaliser la relation avec le client, à mieux le connaître. Les nouvelles technologies ne sont pas étrangères au boom de la fidélisation puisqu'elles ont l'avantage de s'adapter aux clients, d'entretenir une relation à long terme et surtout, d'être interactives (Benavent et Meyer-Waarden, 2004). En d'autres termes, elles rendent possible et dynamisent une stratégie de fidélisation.

\section{A la recherche d'indices de fidélisation}

18 Pour mesurer l'éventuelle implication des programmes ou autres bulletins dans une stratégie de fidélisation, nous avons recensé et analysé les éléments scriptovisuels et les choix en matière médiatique (qui s'inscrivent dans l'énoncé d'une promesse, de l'exposition d'une performance ou qui indiquent que la relation entre l'association et ses membres est personnalisée, interactive et durable).

19 De quelle manière l'association présente-t-elle ses produits? Avec quels arguments? De quel ordre est sa promesse ? Fait-elle la différence entre ses différents publics? 
20 La section suivante reprend les éléments et procédés communicationnels qui font écho à une éventuelle intention de fidéliser les adhérents et qui sont largement partagés par la quinzaine d'associations.

\section{Communiquer pour fidéliser aux activités culturelles ?}

Le discours est axé sur le registre de la qualité et de la performance. L'intention pourrait donc être celle de conserver les adhérents en rendant évidente la qualité des services proposés. En somme, la communication nous donnerait à voir de belles promesses.

\section{Du sur-mesure culturel}

Première observation, l'importance de la diversification des services culturels. Celle-ci prend logiquement toute son ampleur à la lecture du programme. Il est adressé aux adhérents pour qu'ils se fassent usagers, "consommateurs de culture ». Il est par nature le « lieu » où s'énonce l'éventail des produits culturels proposés par l'association. Mais cet éventail de services produit un effet de surabondance tout à fait intéressant.

Les associations d'amis de musées ${ }^{6}$ y présentent très précisément chaque activité : contenu, animateur, dates de déroulement, lieu, résumé du contenu. Le programme expose une diversité telle qu'il ressemble parfois à un catalogue publié par une agence de voyages. Il est la preuve de l'intention de faire du sur-mesure pour les usagers. Chez les professionnels du tourisme, cette surabondance de propositions est d'ailleurs l'un des indices de la personnalisation de masse des voyages (Pelé-Lecorvec, 2003), personnalisation dont on sait qu'elle est un moyen de fidéliser le client (Noyé, 2004).

Les Amis des musées de Chambéry proposent pour la saison 1999-2000 non moins de 32 pages et 11 activités différentes : voyages de durées variables, ateliers à destination de publics différents, cycles de conférence sur plusieurs thèmes... Le lecteur ne peut que se sentir étourdi par la quantité des activités proposées.

Ce faisant, ces associations se dévoilent comme de véritables organisations professionnelles puisqu'elles savent faire la distinction entre les différents publics de l'association et leur proposer des services adéquats. Ainsi, les Amis des musées de Chambéry, du musée d'art moderne de Saint-Etienne, de Brou, du musée de Valence, etc. proposent des activités spécifiquement adressées au jeune public et l'annoncent dans tous leurs outils de communication. En insistant sur cette diversité, l'association souhaite peut-être démontrer qu'il n'y a pas lieu pour l'adhérent de quitter l'association.

26 Cela est d'autant plus évident que ce groupe d'associations use d'une stratégie iconographique qui consiste à mettre en scène le public en train de "consommer » des activités culturelles et d'une certaine manière, à impliquer le lecteur et à le mettre en confiance. On peut se demander si, lorsque les Amis de musée de Brou mettent en scène les usagers en visite, en conférence... ils ne visent pas à témoigner du succès de leurs activités.

En s'exposant à nous comme une véritable entreprise culturelle, l'association ne vise pas l'adhérent mais l'usager des services culturels, c'est-à-dire celui qui, au final, adhère plus pour les avantages liés à l'adhésion (accès aux activités et souvent à des tarifs préférentiels) que pour être membre actif ou seulement pour soutenir le musée. Il s'agit 
alors d'un abonné amateur d'art, d'histoire... plus que le fidèle à l'association ou au musée.

Dans un second temps, l'association insiste sur la valeur ajoutée liée à la «consommation» des services culturels. Cette valeur ajoutée passe par l'accompagnement de l'adhérent dans le choix de ses activités.

\section{Gestion de la relation : encadrement et interactivité}

Il semble que tout soit mis en place, d'un point de vue communicationnel tout au moins, pour que les adhérents intéressés par les activités soient encadrés dans leur démarche, depuis la prise de connaissance des activités jusqu'aux modalités d'inscription aux activités.

Cet encadrement s'accompagne d'une certaine «dose» d'interactivité faisant partie intégrante d'une gestion de relation optimum (Bénavent et Meyer-Waarden, 2004).

31 Cette dernière est rendue possible par la fréquence de la publication des bulletins (en général, 2 à 4 par an) et des programmes (d'une périodicité annuelle à trimestrielle). L'association tient régulièrement au courant ses adhérents des possibilités d'activités, voire, leur donne la possibilité de les conseiller dans leurs choix en fournissant les coordonnées des personnes à contacter ou un descriptif de l'activité.

Cette interactivité occasionnée par les outils de communication démontre l'importance, pour les associations, de gérer la relation à l'adhérent.

Les Amis du musée de Grenoble diffusent en 2000 un calendrier permettant de repérer mois après mois les dates des voyages, les dates d'inscription à ces voyages et les personnes à contacter. Il s'agit pour l'adhérent d'organiser la préparation de son programme individuel en toute sécurité puisque la démarche est balisée : contenu, lieu et date de l'activité... Ce programme a une forme attractive, il renforce le lien entre l'association et l'usager en permettant à ce dernier d'améliorer la gestion dans le temps de ses activités.

34 L'association devance les questions des usagers et des futurs usagers et montre que la relation est interactive et non unilatérale. "Fidéliser un client implique de le conseiller, être réactif et proactif» (Lacroix-Sabalayrolles, 2002) indique une spécialiste. Les outils de communication nous donnent cette impression.

Le troisième « ingrédient » de la valeur ajoutée est la qualité des prestations.

\section{Qualité et satisfaction}

Le registre de la qualité est certainement ce qui est le plus frappant. En effet, lorsqu'elle se met en scène, l'association d'amis de musées nous fait clairement la promesse d'un service de qualité. Ce registre est commun à tous les documents. Pour la plaquette, en tant que document d'appel, il n'y a rien d'étonnant: sa fonction est de convaincre le lecteur d'adhérer. Mais les programmes et bulletins s'engouffrent eux aussi dans une autocélébration. Plusieurs procédés communicationnels cohabitent. 


\section{Crédibilité scientifique}

37 Le premier a pour effet de crédibiliser l'association. Il passe par la mise en avant des qualités professionnelles et intellectuelles des conférenciers et autres intervenants. Chacun est présenté à travers son curriculum vitae indiquant les titres universitaires, les travaux conduits par celui-ci. C'est ainsi que «conservateurs ", « animateurs qualifiés » ou autres « universitaires » interviennent. Leurs compétences universitaires et pédagogiques sont mises en avant pour témoigner que l'exigence est au rendez-vous des activités. Ce procédé permet aussi de prendre la mesure du carnet d'adresses des associations : les Amis des musées de Chambéry invitent par exemple Robert Badinter à la conférence inaugurale de la saison 1999-2000. La qualité côtoie ici l'exceptionnel du service proposé.

\section{Rhétorique de l'autocélébration} travers des indications de durée ${ }^{10}$. L'association s'inscrit dans la durée, ce qui renforce le côté qualitatif de ses actions, et de ses choix stratégiques, rassure l'usager sur les activités culturelles et le conforte dans ses choix. En premier lieu, les associations proposent très souvent des activités sur l'ensemble de la saison, ce qui implique le sens de l'anticipation et de la gestion. En second lieu, certains outils de communication valorisent l'ancienneté de l'association et par-là même, insistent sur les savoir-faire associatifs. Les Amis du musée de Grenoble ou les Amis de Brou ont édité une plaquette pour célébrer leurs 10 ans. Celle-ci retrace l'histoire de l'organisation (contexte et objectif de la création, présentation des fondateurs...) mais souligne aussi les compétences associatives puisque la durée de vie de l'organisation s'explique par ces talents. L'association iséroise y accumule les chiffres : " 15400 adhérents, 243 conférences, 32 journées culturelles... » Dans son programme 1999-2000, les Amis du musée de Valence indique : "Notre association a un an d'existence, une année de travail qui a porté ses fruits puisque 390 adhérents sont venus nous rejoindre " laissant ainsi entendre que l'intensité des actions reste très soutenue et que cette cadence est loin d'être terminée. La manifestation des compétences associatives prend plusieurs tours : exposé des qualités d'organisation, inscription dans la durée ou diversité des services proposés. Cet ensemble de procédés communicationnels dresse des 
passerelles selon nous avec des procédés proches de ceux auxquels les entreprises ont recours pour attirer et fidéliser leurs clients.

Ainsi, les programmes et autres bulletins démontrent bien que le risque lié à la « consommation » d'une conférence est proche de zéro ; ils mettent en valeur les attributs des services culturels (qualité, quantité...). En communiquant de la sorte, l'association d'amis de musée pourrait donc s'engager sur le terrain de la fidélisation active, à savoir, répondre durablement aux attentes des amateurs d'histoire de l'art, d'histoire naturelle, etc. Mais alors, pourquoi la communication des associations contient-elle des éléments nous laissant à penser à une stratégie de fidélisation? Et avant tout, s'agit-il bien d'une stratégie de fidélisation?

\section{Une intention, en apparence}

42 En réalité, plutôt que de parler de l'application communicationnelle d'une stratégie communicationnelle visant à fidéliser les adhérents, nous préférons plutôt évoquer l'existence de quelques ressemblances.

\section{L'effet d'une marchandisation culturelle?}

Cette «impression » de fidélisation provient en premier lieu d'une question essentielle pour les spécialistes du monde associatif (qu'ils soient sociologues, politologues ou spécialistes des SIC): la "contamination» du monde non-lucratif par celui de l'entreprise. N'opère-t-on pas trop rapidement un rapprochement entre les politiques associatives et celles des entreprises?

Certains auteurs établissent des passerelles entre la communication des entreprises et celle des organisations non lucratives, notamment dans le domaine social (Meyer, 2004), humanitaire (Lavoinne, 2002) ou en général (Dacheux, 2001). Cela va du constat de la professionnalisation des actions de communication à l'évocation de la perte de la spécificité et des valeurs liées au modèle associatif. Cette tendance est souvent interprétée comme le signe de la modernité associative. La fidélisation pourrait être une des conséquences de cette évolution et transparaître dans la manière de communiquer.

Mais à se pencher sur les raisons d'un éventuel développement de politique de fidélisation dans la sphère associative, on s'aperçoit que les explications de ces « effets de communication" ne proviennent pas du secteur lucratif, mais selon nous, de deux processus distincts.

\section{La pression des partenaires}

Quel est l'intérêt pour les amis de musées de donner l'impression de qualité et de susciter en nous l'impression d'une stratégie de fidélisation? En s'inscrivant dans la perspective interactionniste de Goffman on peut comprendre que la «communication illustre une contrainte institutionnelle qui pèse sur l'acteur et transforme ses activités en représentation" (1973 : 67). De ce fait, cette manière de communiquer est pour l'organisation, davantage l'effet de la volonté de donner une impression à ses partenaires: le musée et ses institutions de tutelle (les collectivités locales et l'Etat) et moins une stratégie envers ses adhérents. Nous pensons que la rhétorique de légitimation et de crédibilisation qui 
consiste à témoigner et à insister sur les compétences de l'association sert à attester du processus de professionnalisation qui touche le secteur associatif. Meyer observe la rhétorique professionnelle des associations du secteur social (Meyer, 2004). L'engagement de ces associations devient une "vitrine de la professionnalité» (Meyer, 2004: 38), une occasion de dévoiler les compétences et qualités en externe et en interne.

La volonté de se crédibiliser est d'une part à destination du musée qui se professionnalise lui-même. Les associations souhaitent prouver qu'elles possèdent toujours une place dans le monde muséal; porter attention à l'adhérent devient donc le pendant de l'attention portée par le musée à ses publics. La nécessité de se rendre crédible est d'autre part une réponse aux exigences de l'Etat qui souhaite s'adresser à des partenaires professionnels. D'où la nécessité pour les associations en charge du service d'animation de montrer leurs compétences lorsqu'elles communiquent. Il en va des subventions accordées !

\section{L'effet de l'évolution de l'engagement}

48 Le second processus que rend visible notre questionnement est celui de l'évolution de l'engagement. En effet, Barthelemy (2000) et Ion $(2001,2003)$ ont démontré qu'une des transformations du monde associatif était l'émergence d'un « militantisme affranchi » (Ion, 2003), c'est-à-dire témoignant de savoirs pointus plutôt que d'un projet politique, à la carte plutôt, que de longue durée. La montée en puissance de la satisfaction des besoins des membres prend le pas sur le militantisme politique, dévoilant ainsi le boom des associations autocentrées (Ion, 2001). La communication des associations d'amis de musées illustre parfaitement cette évolution. Cela explique pourquoi les savoirs et les compétences sont valorisés. Mais cela explique aussi pourquoi les associations souhaitent conserver leurs adhérents: leur implication à long terme n'est plus évidente. La communication pourrait venir pallier le papillonnage et emprunter de manière plus ou moins consciente aux techniques du secteur lucratif.

\section{Conclusion}

Malgré les limites de notre réflexion qui sont la petite taille du terrain et l'absence de la prise en compte de l'interprétation des intéressés, plusieurs pistes à approfondir émergent et mériteraient d'être creusées. Nous avons observé que l'association communique moins sur la raison de son existence: le soutien au musée, que sur une approche que l'on pourrait qualifier d'orientée client. Car au final, il serait plus sage de parler d'une tendance à cette orientation que d'une réelle intention de fidéliser le client qui reprend les méthodes éprouvées par les entreprises. L'utilisation de certains médias (périodiques et programmes) et l'emploi de certains procédés communicationnels laissaient à penser que l'association d'amis de musées était préoccupée par la fidélisation des ses adhérents à ses activités. Au terme de cet article, nous pensons que cela est certainement abusif.

Mais l'intérêt est de constater que la seule analyse de la communication permet ici de comprendre que l'adhérent à l'association est une catégorie d'acteurs bien trop neutre. Les manières de s'engager dans le milieu associatif sont très hétéroclites. Dans notre cas, il ressort que la cible visée dans les outils de communication étudiés est plutôt l'usager, l'adhérent bénéficiaire, qui, en tant qu'amateur, est exigeant dans ses choix. De ce fait, la 
frontière entre l'adhérent et le client fidèle usager des activités est ici tenue. Surgit alors de cette confusion l'évocation logique de la fidélisation.

\section{BIBLIOGRAPHIE}

Almeida N. (d'), Les promesses de la communication, Paris : PUF, 2000.

Barthelemy M., Associations, un nouvel âge de la participation, Paris : Presses de Sciences Po, 2000.

Benavent C., Meyer-Waarden L., « Programmes de fidélisation : stratégies et pratiques », in :

Revue française de marketing, $n^{\circ}$ 197, mai 2004, p. 95-115.

Chaumier S., Des musées en quête d'identité, Paris : L'Harmattan, 2003.

Cochoy F., La captation des publics, Toulouse : Presses universitaires du Mirail, 2004.

Dacheux E., «Le marketing à la lumière de la communication » in : L'année sociologique, 51, 2001, p. 411-427.

Darpy D., Volle P., Comportements du consommateur : concepts et outils, Paris : Dunod, 2003.

Davallon J., « L'évolution du rôle des musées » in : Lettre de l'OCIM, n 49, 1997, p. 4-8.

Di Sciullo J., Marketing et communication des associations, Paris/ Lyon : Juris, 1988.

Goffman E., La mise en scène de la vie quotidienne, Paris : Minuit, 1973.

Ion, J., « Modes d'engagement et savoirs associatifs : petit coup d'œil dans le retroviseur », in : Lochard Y. et Simonet-Cusset M., L'expert associatif, le savant et le politique, Paris : Syllepse, 2003, p. 21-26.

Ion, J., « Un engagement en mutation », in : CAES info, $\mathrm{n}^{\circ} 61,2001,4 \mathrm{p}$.

Lacroix-Sablayrolles H., Marketing stratégique orienté client, Paris : Dunod, 2002.

Laville J.-L., « L'association : ni entreprise, ni administration » in : Bloch-Lainé, F., Faire société, Syros, 1999, p. 93-108.

Laville, J.-L., « L'association comme lien social », Sciences Humaines, hors-série, n 33, 2001, p. 64-67.

Lavoinne Y., L'humanitaire et les médias, Lyon : PUL, 2002.

Meyer V., Interventions sociales, communication et médias. L'émergence du sociomédiatique, Paris : L'Harmattan, 2004.

Noyé D., Pour fidéliser les clients, Paris : INSEP Consulting, 2004.

Pelé-Lecorvec G.-N., « Donatello cultive l'art de vivre en liberté » in : Espaces, tourisme et loisirs, $\mathrm{n}$ - 205, juin 2003, p. 34-35.

Sainsaulieu R., « Associations et entreprises », in : Laville J.-L. et Sainsaulieu R.(dir). Sociologie de l'association, Paris : Desclée de Brouwer, 1997, p. 301-316. 
Siméant J. et Dauvin P., «La professionnalisation humanitaire comme réalité et comme enjeu », in : Actions associatives, solidarités et territoires, Actes du colloque organisé par le Crésal, octobre 2001, p. 199-206.

Thevenard-Nguyen C., Les associations d'amis de musées, leur position et leur engagement dans l'espace public, Davallon J. (dir.), université d'Avignon, 2002.

\section{NOTES}

1. C'est en raison des caractéristiques de la médiatisation de leur engagement que nous avons souhaité travailler plus particulièrement sur la communication écrite de ces 15 associations. Cette médiatisation indiquait en effet que ces organisations s'affichaient comme de véritables entreprises d'animation culturelle. Par comparaison, les autres groupes communiquaient de manière revendicative, insistaient sur l'aspect savant; le devoir de mémoire ou la promotion d'un produit local.

2. $37 \%$ des 15 associations loi 1901 de ce groupe utilisent 3 supports de communication (plaquette, programme et bulletin). Dans les autres groupes, aucune des associations n'est dans ce cas.

3. Les effectifs tournent en moyenne entre 100 et 400 membres. Ils sont 1600 au Musée des Beaux-Arts de Grenoble.

4. La FFSAM est née il y a une trentaine d'années et compte 280 adhérents en 2004.

5. Elles ont toutefois la possibilité de se présenter individuellement sur le site de la fédération : www.amis-musees.fr. Ces pages sont la plupart du temps la stricte reprise des plaquettes et programmes.

6. La moitié des associations possède un programme, un tiers publie un périodique (lettre ou bulletin). Dans ces deux cas, le support de communication est envoyé automatiquement aux membres de l'association. Le bulletin présente parfois les activités à venir et reprend le rôle du programme. Deux tiers d'entre elles ont édité une plaquette (les associations cumulent parfois plusieurs outils).

7. Ce terme est très souvent utilisé par les associations.

8. Programme des Amis des musées de Chambéry, 1999-2000.

9. Programme 1997-1998 des Amis du musée de Valence.

10. I. Pailliart traite de cette question dans un article de 1987: «La télématique et les associations », in : La Tribune Fonda, 50, p. 21-29.

\section{RÉSUMÉS}

A partir de l'analyse de la communication d'un ensemble d'associations du secteur culturel (les associations d'amis de musées), nous proposons d'étudier et de questionner leurs intentions de fidéliser et de personnaliser les relations avec les adhérents. Nous verrons que malgré quelques similitudes sur le plan de la communication, l'analogie avec la fidélisation opérée par les entreprises est loin d'être évidente. 
This article tries to answer that question: do societies intend to personalize and develop members' loyalty? Our reflexion is based on the analysis of texts and pictures contained in documents produced by societies (Friends of Museums). We will see that it is not so easy to make a connection between what firms and societies operate.

\section{AUTEUR}

\section{CÉLINE NGUYEN}

Céline Nguyen est docteur en sciences de l'information et de la communication. Elle enseigne à l'INSA de Lyon et est membre de l'équipe pluridiciplinaire STOICA de l'INSA de Lyon et du Laboratoire Culture et Communication de l'Université d'Avignon. Ses recherches actuelles dans le champ de la communication des organisations concernent la communication des associations culturelles et les récits d'instituts de formation. Mail : celine.nguyen@insa-lyon.fr 